\title{
Characteristics of magnetic labeling on liver tumors with anti-alpha-fetoprotein-mediated $\mathrm{Fe}_{3} \mathrm{O}_{4}$ magnetic nanoparticles
}

This article was published in the following Dove Press journal:

International Journal of Nanomedicine

21 June 2012

Number of times this article has been viewed

\author{
Kai-Wen Huang' \\ Jen-Jie Chieh ${ }^{2}$ \\ Herng-Er Horng ${ }^{2}$ \\ Chin-Yih Hong ${ }^{3}$ \\ Hong-Chang Yang ${ }^{4}$ \\ 'Department of Surgery and Hepatitis \\ Research Center, National Taiwan \\ University Hospital, Taipei, ${ }^{2}$ Institute \\ of Electro-optical Science and \\ Technology, National Taiwan Normal \\ University, Taipei, ${ }^{3} \mathrm{Graduate}$ Institute \\ of Biomedical Engineering, National \\ Chung Hsing University, Taichung, \\ ${ }^{4}$ Department of Physics, National \\ Taiwan University, Taipei, Taiwan
}

\begin{abstract}
For preoperative and intraoperative detection of tumor distribution, numerous multimodal contrast agents, such as magnetic nanoparticles (MNPs) with several examination indicators, are currently in development. However, complex materials, configuration, and cost are required for multimodal contrast agents, accompanied by a high possibility of toxicity and low popularity in clinics. Nevertheless, the magnetic labeling of MNPs using bioprobes should be feasible not only in preoperative magnetic resonance imaging (MRI), but also in intraoperative examination based on other magnetic properties. In this study, anti-alpha-fetoprotein (AFP)-mediated $\mathrm{Fe}_{3} \mathrm{O}_{4} \mathrm{MNPs}$, injected into mice with liver tumors, were used to examine the characteristics of magnetic labeling. Using MRI and scanning superconducting-quantum-interference-device biosusceptometry (SSB), based on alternating current (AC) susceptibility, the magnetic labeling occurred significantly on the first day post-injection of anti-AFP magnetic fluid (MF), and then decreased over time. However, for both MF without antibodies and an anti-carcinoembryonic antigen MF, no magnetic labeling occured on the first day of their respective post-injection. The favorable agreement indicates that magnetic labeling possesses two magnetic characteristics: distortion of the imaging field and $\mathrm{AC}$ susceptibility. In addition, the results of the biopsy tests, anti-AFP staining, and Prussian blue staining show the same dynamics as those of magnetic methodologies and prove that bound MNPs on tumor tissue are rotatable by an AC magnetic field to express AC susceptibility. Therefore, with the simple configuration of antibody-mediated MNPs, magnetic labeling is also feasible for intraoperative examinations using SSB with high mobility and sensitivity.
\end{abstract}

Keywords: alpha-fetoprotein, magnetic resonance imaging, scanning SQUID biosusceptometry

\section{Introduction}

The superior magnetic characteristics of bioprobe-mediated magnetic nanoparticles (MNPs) have attracted attention for application in biomedicine and nanomedicine fields, such as image contrast, ${ }^{1}$ immunoassay immuno-magneto-reduction (IMR), ${ }^{2,3}$ hyperthermia, ${ }^{4,5}$ drug delivery, ${ }^{6,7}$ and surgery. ${ }^{8}$ Among such fields, numerous magnetic methodologies, therapy, and instruments have been developed to process these various functions. However, a number of limitations remain in the current technologies for each topic.

For example, with bound MNPs as the image contrast to improve the diagnosis specificity of magnetic resonance imaging (MRI), MRI remains difficult to be popularized because of its high cost and maintenance, especially when applied as an intraoperative examination based on concerns regarding surgical resection of tumors. ${ }^{9}$

Multimodal nanoparticles, ${ }^{9,10}$ such as a bioprobe-mediated MNP with an extra fluorochrome conjugation, were developed to track the same nanoparticles using 
both preoperative MRI and intraoperative methodologies such as optical imaging. However, the complex synthesized technology usually limits popularity in clinics, and the uncertainty of toxicity and stability characteristics might increase.

Examination of the simple MNPs, the key topic of magnetic labeling, should be studied for preoperative or intraoperative examination. For example, the principle of bound MNPs as image contrasts of the preoperative MRI is that the distortion of the excitation field is induced from MNPs or numerous iron ions of biodegraded MNPs (Figure 1). ${ }^{11}$ Therefore, tumors labeled by bound MNPs can be identified by darkening the image brightness. This procedure is especially valuable for the preoperative diagnosis of liver tumors because of the complexity of liver tissue. ${ }^{12}$ In addition, the principle of targeting hyperthermia shows the out-of-phase alternating current (AC) susceptibility of bound MNPs under high $\mathrm{AC}$ strength and frequency of magnetic field results in the generation of heat power. ${ }^{4}$ This indicates that AC susceptibility of bound MNPs under low AC strength and frequency of the magnetic field could be adopted for another examination characteristic without the possibility of heat generation. This characteristic has recently been proven for free MNPs in animals using scanning superconducting-quantum-interferencedevice (SQUID) biosusceptometry (SSB). ${ }^{13-15}$ The feasibility for detecting bound MNPs on tumors has yet to be tested. If such a procedure is effective, SSB could be used as an intraoperative instrument because SSB possesses the advantages of high sensitivity of a SQUID sensor, high examination mobility around the torso, easy and practical operation, and high safety and more cost-effectiveness of low AC-field excitation. SSB has greater potential for popularization.

In this work, magnetic labeling on the liver tumors of mice with the injection of anti-alpha-fetoprotein (AFP)-mediated

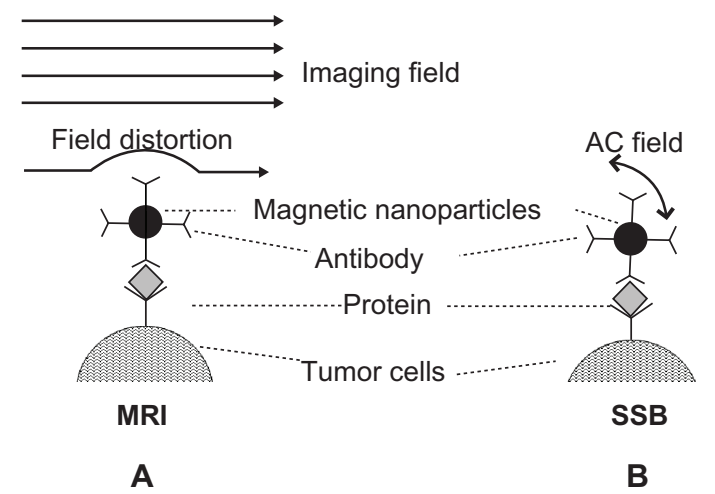

Figure I Illustration of mechanism of (A) MRI and (B) SSB examination for antibody-mediated magnetic nanoparticles on liver tumor tissue.

Abbreviations: AC, alternating current; MRI, magnetic resonance imaging; SSB, scanning superconducting-quantum-interference-device biosusceptometry.
MNPs was examined using MRI and SSB. Furthermore, the biopsy test was conducted to illustrate the magnetic results. To prove that the magnetic labeling resulted from the specific binding between MNPs and tumor tissue, MNPs without an antibody coating and with an anti-carcinoembryonic antigen (CEA) coating were also processed.

\section{Materials and methods}

The Animal Care and Use committee of the College of Medicine, National Taiwan University, approved all experimental protocols (No 20110009). All experiments were conducted in accordance with the animal care guidelines of the university.

\section{Tested animals and tumor materials}

For 8-week-old BALB/C mice (Laboratory Animal Center, National Taiwan University, Taipei, Taiwan, Republic of China), the injections of the BNL-1ME-A.7R.1 (BNL) cell line were processed through the back skins of five mice to plant the liver tumors. In this study, BNL, a mouse liver adenocarcinoma cell line (ATCC, Manassas, VA), was maintained in Dulbecco's Modified Eagle's Medium supplemented with $10 \%$ heat-inactivated $\left(56^{\circ} \mathrm{C}, 30\right.$ minutes $)$ fetal bovine serum, antibiotics, and antimycotics (Life Technologies, Inc, Gaithersburg, $\mathrm{MD}$ ) in a humidified atmosphere of $5 \% \mathrm{CO}_{2}$ at $37^{\circ} \mathrm{C}$. The BNL cells expressing AFP is described as follows: The BNL cells were stably transfected with pBBS212-hAFP eukaryotic expression vectors through calcium phosphorate precipitation (Promega Technologies, Inc, Madison, WI). Positive cell clones were screened using a conditioned medium, and supernatants were detected using an AFP radioimmunoassay, following manufacturer instructions. Details of the experiments performed on these mice are shown in Table 1.

\section{Magnetic fluids}

Three types of magnetic fluids (MFs), anti-AFP $\mathrm{MF},{ }^{3} \mathrm{MF}$ without antibodies (MF-DEX-0060, MagQu, New Taipei, Taiwan, Republic of China), and an anti-CEA MF, were used (inset of Figure 2A). The MF without antibodies was composed of a water solution and $\mathrm{Fe}_{3} \mathrm{O}_{4}$ MNPs coated by dextran. For the used anti-AFP MF, the dextran of the MF was oxidized with $\mathrm{NaIO}_{4}$ to create aldehyde groups $(-\mathrm{CHO}) .{ }^{16} \mathrm{The}$ dextran then reacted with the anti-AFP through $-\mathrm{CH}=\mathrm{N}-$ to covalently conjugate anti-AFP antibodies (EA502-Q1053; EastCoast Bio, North Berwick, ME, USA) onto MNPs. Through magnetic separation, the unbound antibodies were separated from the magnetic reagent. Similarly, for the used anti-CEA MF, the synthesis process was identical, but involved 
Table I Information of in-vivo tests and biopsy tests in this work

\begin{tabular}{|c|c|c|}
\hline Reagent & In-vivo tests & Biopsy tests \\
\hline \multirow[t]{7}{*}{ Anti-AFP MF } & MF dose: $0.06 \mathrm{emu} / \mathrm{g}, 100 \mu \mathrm{L}$ & Sacrifice time \\
\hline & Mouse I: at 0,13 , and 20 hours (by SSB) & Mouse I: at 26 hours \\
\hline & at 0 and 24 hours (by MRI) & Mouse 2: at 50 hours \\
\hline & Mouse 2: at $0,17,20$, and 44 hours (by SSB) & Mouse 3: at 74 hours \\
\hline & at 0 and 48 hours (by MRI) & Stain kinds \\
\hline & Mouse 3: at $0,4,11,24,37,48$, and 68 hours (by SSB) & HE stain, Prussian blue stain, anti-AFP stain \\
\hline & at 0 and 72 hours (by MRI) & \\
\hline \multirow[t]{4}{*}{ MF without antibodies } & MF dose: $0.06 \mathrm{emu} / \mathrm{g}, 100 \mu \mathrm{L}$ & Sacrifice time \\
\hline & Mouse 4: at 0 and 20 hours (by SSB) & Mouse 4: at 26 hours \\
\hline & at 0 and 24 hours (by MRI) & Stain kinds \\
\hline & & HE stain, Prussian blue stain \\
\hline \multirow[t]{4}{*}{ Anti-CEA MF } & MF dose: $0.06 \mathrm{emu} / \mathrm{g}, 100 \mu \mathrm{L}$ & Sacrifice time \\
\hline & Mouse 5: at 0 and 20 hours (by SSB) & Mouse 5: at 26 hours \\
\hline & at 0 and 24 hours (by MRI) & Stain kinds \\
\hline & & HE stain, Prussian blue stain \\
\hline
\end{tabular}

Abbreviations: AFP, alphafetaprotein; CEA, carcinoembryonic antigen; HE, hematoxylin and eosin; MF, magnetic fluid; MRI, magnetic resonance imaging; SSB, scanning superconducting-quantum-interference-device biosusceptometry.
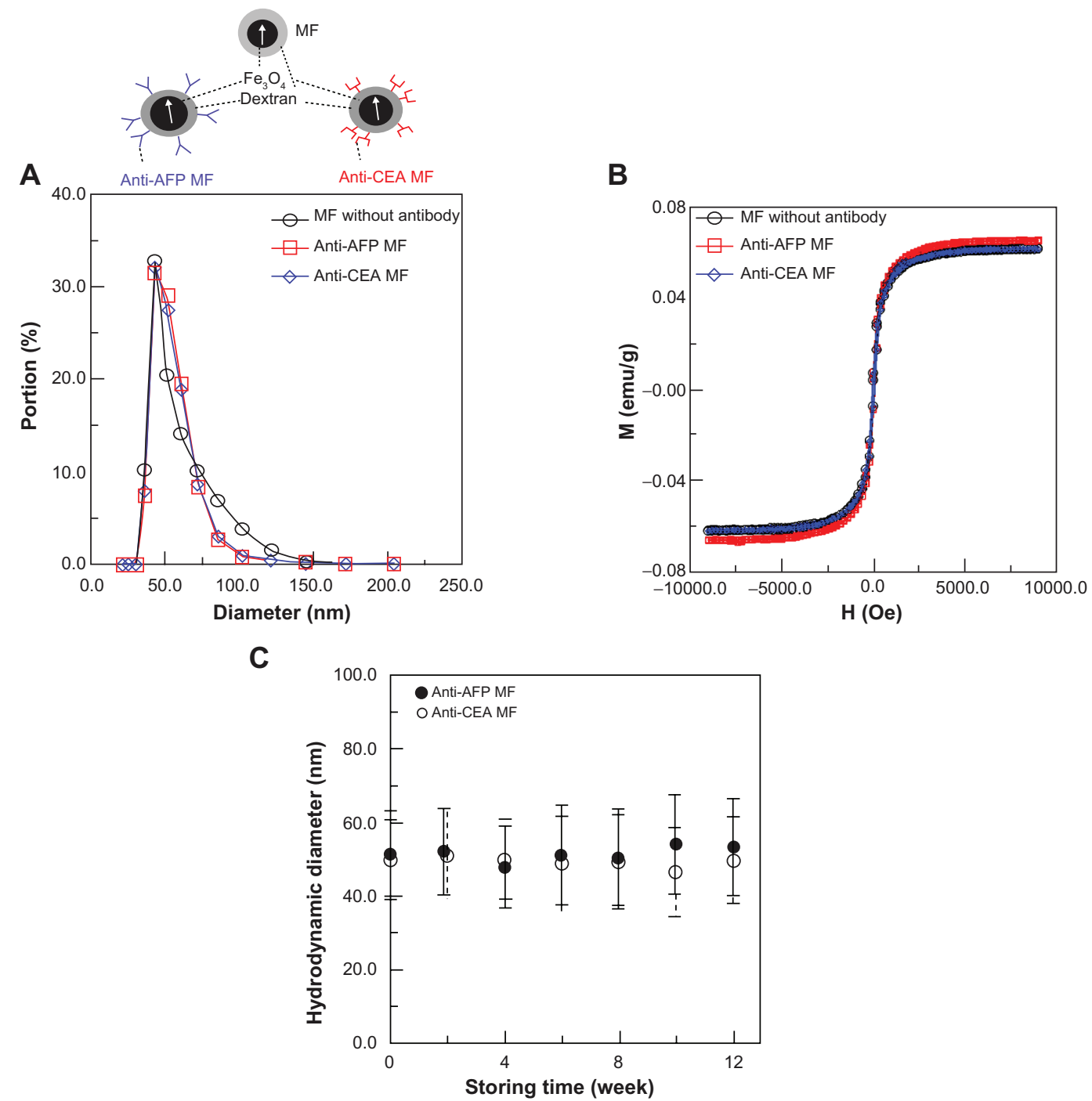

Figure 2 Characteristics of anti-CEA MF, anti-AFP MF, and MF without antibody coatings: (A) the hydrodynamic diameters, (B) saturation magnetism, (C) the stability of antibody-mediated MFs.

Abbreviations: CEA, carcinoembryonic-antigen; MF, magnetic fluid; AFP, alphafetaprotein. 
using an anti-CEA antibody (10C-CR2014M5, Fitzgerald, MA) instead of an anti-AFP antibody. The objective of this study was to verify that magnetic labeling using bound MNPs possesses higher AC susceptibility for examination. The magnetic labeling of MFs without antibodies, which were transported rather than bound to the tumor tissue, was used as a reference of free MNPs and compared with anti-AFP MNPs. Furthermore, the magnetic labeling of MNPs with specific binding rather than a biological attachment to the tumor tissue also required verification. Magnetic labeling of anti-CEA MF, which is nonspecifically attached to liver tumors, was compared with that of anti-AFP MF, which is specifically bound to tumors. Here, the choice of anti-CEA MF was based on a radiolabeling comparison between antiCEA and anti-AFP antibodies coated on iodine. ${ }^{17}$ Afterward, both anti-CEA MF and anti-AFP MF were verified to have similar AC susceptibility for binding with their specific plasma antigens by using in-vitro IMR tests. ${ }^{18}$

\section{Characterization of MFs}

Because the self-aggregation of antibody-mediated MFs reduced their ability to bind to specific antigens in animals, their particle size distributions were measured using a nanotrac particle size analyzer (Microtrac, Montgomeryville, PA) for up to 3 months, and more than 3 days of magnetic labeling to verify their biofunctionality.

In addition, the magnetism of the three MFs was measured using a vibrating sample magnetometer (EG\&G PARC, Newnan, GA) to prove the independence of antibody mediation in the synthesis of MFs on the magnetic characteristics. To prove the feasibility of magnetic labeling using anti-AFP $\mathrm{MF}$, the preliminary in-vitro IMR tests ${ }^{3}$ were processed under AC magnetic fields by using an IMR analyzer (XacPro-E; MagQu, New Taipei City, Taiwan, Republic of China). The results showed a high specificity of anti-AFP without interference from other plasma proteins. ${ }^{3}$

\section{In-vivo tests}

The anti-AFP MF, MF without antibodies, and anti-CEA MF were injected separately into the tail veins of 14-week-old mice with liver tumors (Table 1), using the same injection dose of $0.06 \mathrm{emu} / \mathrm{g}$ and $100 \mu \mathrm{L}$. Here, the concentration of the three MFs injected in this experiment was $10^{-11} \mathrm{M}$, which is significantly lower than the nontoxicity criteria; that is, $10^{-6} \mathrm{M}$ of the pure MNPs coated with liposome in the preliminary test. ${ }^{19}$ Therefore, no possibility of normal and tumor tissue toxicity existed in this work, because the concentration within animals was diluted. To examine the magnetic characteristics of magnetic labeling, the in-vivo tests were processed using developed scanning SQUID biosusceptometry (SSB) $)^{13-15}$ based on AC susceptibility of samples and MRI based on the distortion of the imaging field that resulted from MNPs. ${ }^{11}$ Following standard procedures, the test mice in these in-vivo tests were anesthetized using inhalation anesthesia. In addition, in-vivo examination using SSB was early and more frequent than that using MRI because of expedient and easy operation. The examination time of each mouse is shown in Table 1 , where 0 hours represents the time before injection.

In the MRI examinations, a 7-T MRI system (Bruker, Ettlingen, Baden-Württemberg, Germany) was used for $\mathrm{T}_{2}$-weighted coronal images with an interval of $1 \mathrm{~mm}$. An inhalation anesthesia system (Protech, Boerne, TX), an MRI-compatible real-time physiological monitoring system (Biopac, Goleta, CA), and a circulation system of warm water (Chinchi-tech, Taipei, Taiwan, Republic of China) were used to control the test mice under anesthesia. Aside from the test mice, a tube $4 \mathrm{~mm}$ in inner diameter and $7 \mathrm{~cm}$ in length was filled with deionized water and attached in the sample holder as that depicted in the image reference. Therefore, the analysis of the normalized intensity of the tumor (Figure 3A), defined as the ratio of the average intensity of the tumors marked by blue outlines over that of the reference water marked by blue outlines, was used to suppress the instrument drift at different examination times. Furthermore, all of the normalized intensities of the tumor appearing in MRI coronal images at each designated time were analyzed as the average intensity $I$ for the quantitative analysis of the whole tumor. In addition to the intensity variation of a single tumor region, the image contrast, defined as the ratio of the normalized intensity of the tumor marked by blue outlines over that of the neighboring normal tissue marked by red outlines, was also analyzed. Additionally, all MRI coronal image contrasts of the tumor at the designated times were analyzed as the average image contrast $C$ for a quantitative comparison between the tumor tissue and neighboring normal tissue.

During the SSB examination, the backs of the mice were covered with a thick plastic plate using a tumor-fit hole (Figure 4A) to maintain the same height and tumor orientation as the reference to the scanning path of the scanning probe, which was composed of a pickup coil and excitation coil. This installation method facilitated suppressing the error of the distance between the SSB scanning probe and the tumor on the backs of the mice to within $10 \%$. The scanning speed was $0.5 \mathrm{~mm} / \mathrm{s}$ for each step, over 15-30 mm, depending on the tumor size. Because both the peak amplitude and the width 
A
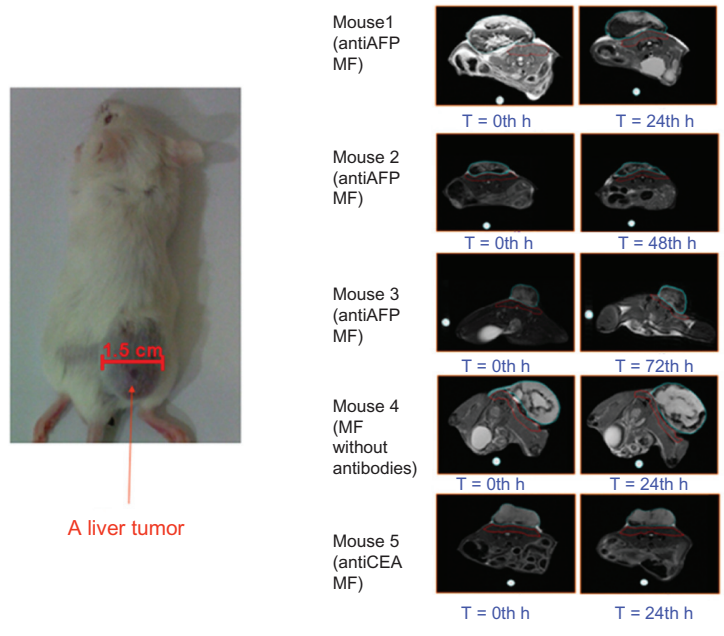

B

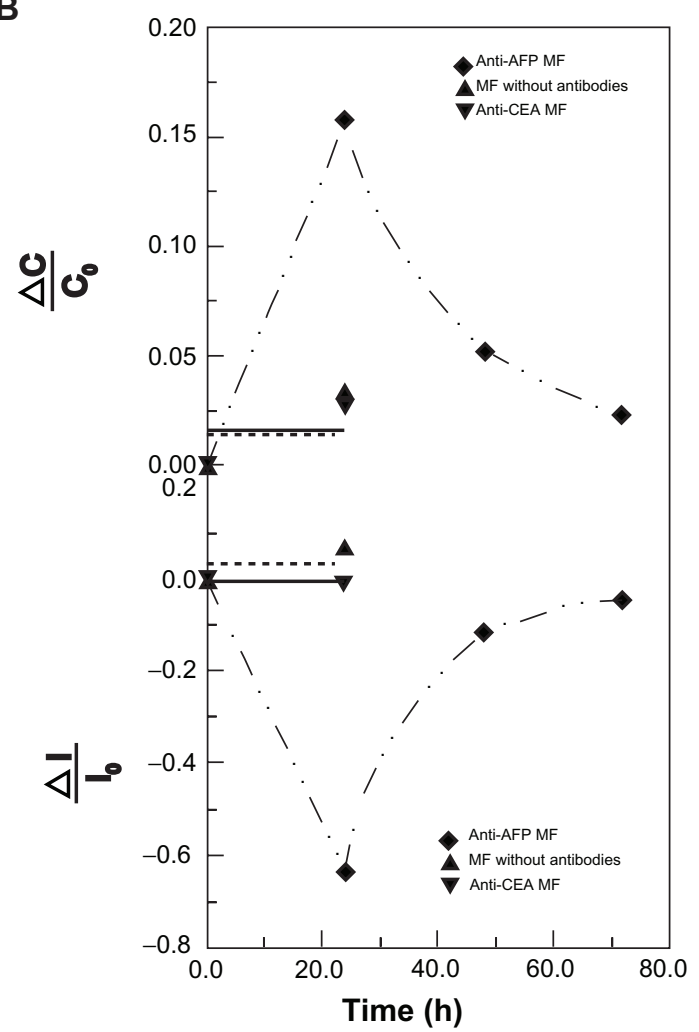

Figure 3 MRI examination of a liver tumor. (A) Photos of the test mice and representative MRI images of the liver tumor at different examination time. (B) The variation ratio of the average normalized intensity $\Delta \mathrm{I} / \mathrm{I}_{0}$ and the variation of image contrast $\Delta \mathrm{C} / \mathrm{C}_{0}$.

Abbreviations: AFP, alphafetaprotein; CEA, carcinoembryonic-antigen; MF, magnetic fluid; MRI, magnetic resonance imaging.

of the scanning curve depend on the sample magnetism $\mathrm{M},{ }^{13}$ at the same distance between the sample and the probe, a scanning area, defined by the product of the scanning interval and the summation of the intensity, was used for magnetism analysis. However, in this study, a reliable scanning area (ie, the product of the modified multiplier of the intensity larger than half the maximum intensity of the scanning curve) was used for SSB analysis, with a repeatability error smaller than $10 \%$.

\section{Biopsy test}

To illustrate the relationship between bound MNPs and magnetic labeling examined using these different magnetic methodologies, hematoxylin and eosin (HE) staining, Prussian blue staining, and anti-AFP staining were used for the liver tumors of euthanized mice, with the injection of different MFs at different times (Table 1). The biopsy test was processed (Laboratory Animal Center, National Taiwan University, Taipei, Taiwan, Republic of China), and the $400 \times$ magnification of the optical images was observed using light microscopy.

\section{Results and discussion Characterization of MFs}

For these MFs, the hydrodynamic diameters of MNPs, as well as the MNPs with anti-AFP and anti-CEA coating, are $57.3 \pm 15.2 \mathrm{~nm}, 54.3 \pm 10.1 \mathrm{~nm}$, and $54.4 .0 \pm 10.5 \mathrm{~nm}$, respectively (Figure 2A). The measured saturation magnetisms of these three magnetic reagents are $0.063 \mathrm{emu} / \mathrm{g}, 0.062 \mathrm{emu} / \mathrm{g}$, and $0.062 \mathrm{emu} / \mathrm{g}$ (Figure 2B). These results show that the three MFs possessed similar hydrodynamic diameters and magnetism, but different biofunctions. Controlling all the same properties except the biofunctions is critical for comparing the magnetic labeling in in-vivo tests between different MFs. Furthermore, antibody-mediated MFs, such as anti-AFP MF and anti-CEA MF, show that their hydrodynamic diameters remained the same for 3 months (Figure 2C), thus proving the high stabilities of these MFs. In other words, if antibody-mediated MFs possess the high stability of particle size, the variation of IMR signals in in-vitro tests ${ }^{3}$ and the magnetic labeling effects in in-vivo tests in this study resulted from the conjugation between MNPs and antigens, rather than from the self-conjugation between MNPs.

\section{In-vivo tests of MFs}

In MRI images with tumor appearance (Figure 3A), the tumor brightness became significantly darker at 24 hours post-injection of anti-AFP MF, and the variation levels decreased over time. To verify that the variation of tumor brightness resulted from the magnetic labeling of anti-AFP MNPs binding to liver tumor tissue, the liver tumors of the mice injected with MNPs without antibodies and anti-CEA MNPs were also observed at 24 hours, which is the most sig- 
nificant magnetic labeling for anti-AFP MNPs. Conversely, little variation was observed, confirming the high specificity of anti-AFP MF in in-vivo tests.

The variation ratio of the average normalized intensity $\Delta \mathrm{I} / \mathrm{I}_{0}$ was used for quantitative analysis of the variation of MRI signals with the suppression of individual difference (Figure 3B). In this study, $\mathrm{I}_{0}$ and I were the average intensity of the tumor before and after the injection, respectively, and $\Delta \mathrm{I}$ was the difference between $\mathrm{I}$ and $\mathrm{I}_{0}$. The trend of antiAFP MF shows that the variation reached the maximum
$\Delta \mathrm{I} / \mathrm{I}_{0}$ at approximately 24 hours, and decreased to its initial level over time. However, the variation of anti-CEA MF and MF without antibodies sustained nearly the same levels, at 24 hours, of the most significant magnetic labeling, indicating that magnetic labeling on the liver tumors for MRI was feasible using only anti-AFP MF. Similarly, the variation of image contrast $\Delta \mathrm{C} / \mathrm{C}_{0}$ was employed to analyze the impact of magnetic labeling using these MFs (Figure 3B). $\mathrm{C}_{0}$ and $\mathrm{C}$ represent the average image contrasts between the tumor tissue and neighboring normal tissue before and after the

A
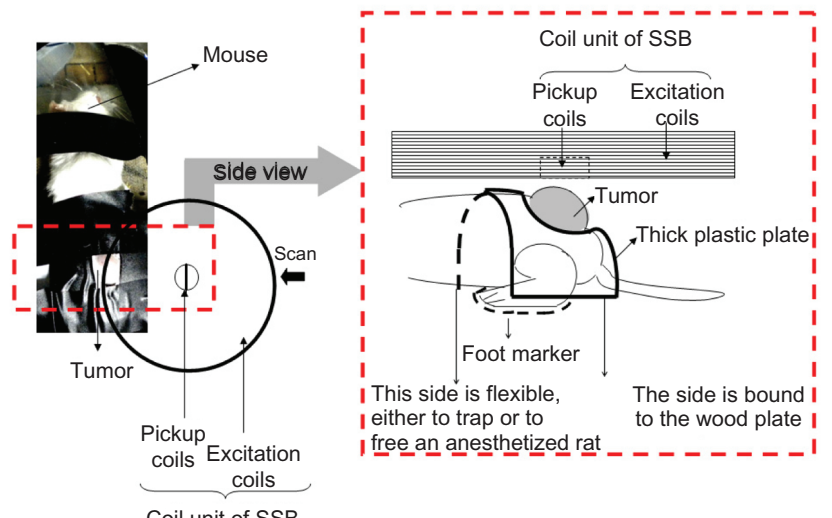

Coil unit of SSB
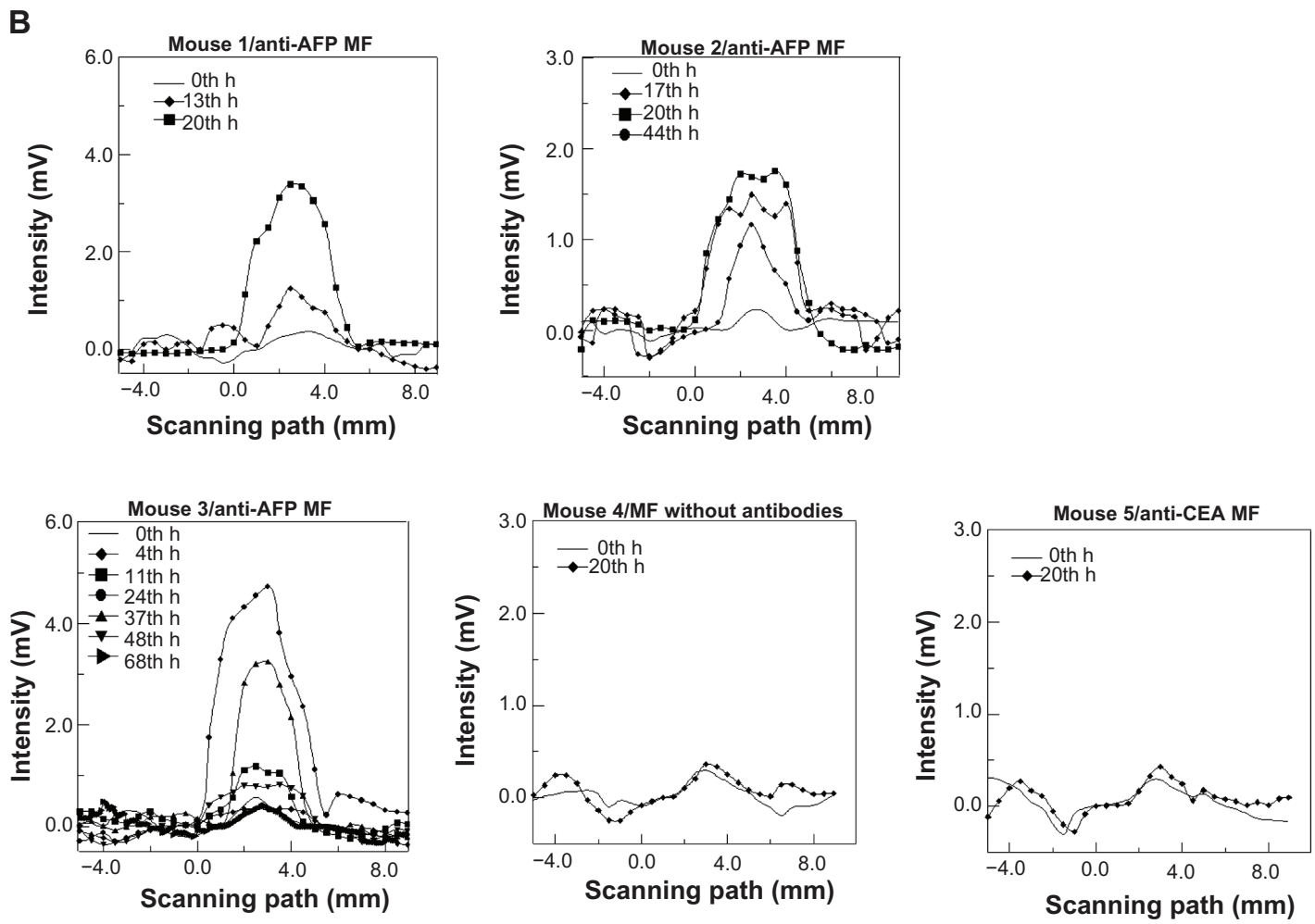

Figure 4 (Continued) 

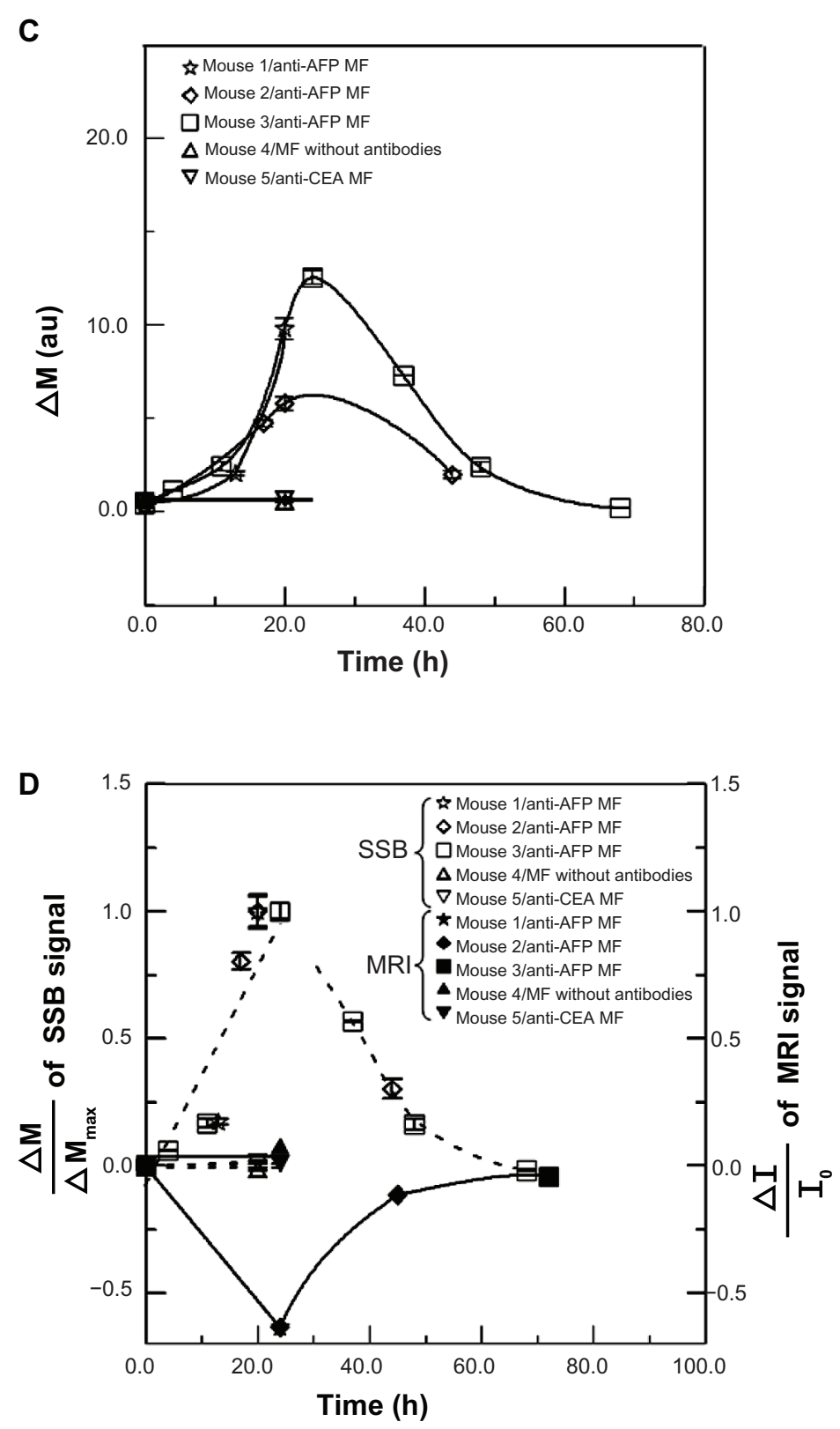

Figure 4 SSB examination of a liver tumor. (A) Setup scheme. (B) The scanning curves of all test mice at different times. (C) The variation of magnetism M of all test mice at different times. (D) The analysis comparison of SSB and MRI.

Abbreviations: AFP, alphafetaprotein; CEA, carcinoembryonic-antigen; MF, magnetic fluid; SSB, scanning superconducting-quantum-interference-device biosusceptometry; MRI, magnetic resonance imaging.

injection, respectively. $\Delta \mathrm{C}$ is the difference between $\mathrm{C}$ and $\mathrm{C}_{0}$. The good agreement between the trends of $\Delta \mathrm{C} / \mathrm{C}_{0}$ and $\Delta \mathrm{I} / \mathrm{I}_{0}$ for the MFs showed that the image contrast resulted from the magnetic labeling.

The scanning curves of the tumor examinations at different times are shown in Figure 4B. The variation of magnetism $\mathrm{M}$, obtained from the reliable scanning area, among three mice injected with anti-AFP MF, showed favorable agreement because $M$ increased to the maximum value at approximately 24 hours, and then decreased over time (Figure 4C). However, $\mathrm{M}$ of mice injected with
anti-CEA MF and MF without antibodies showed seldom variation around 24 hours. This finding indicates that the anti-AFP MNPs were gradually accumulated and bound to the liver tumor tissue until approximately 24 hours, and then departed or biodegraded over time. However, the anti-CEA MNPs and MNPs without antibodies were barely bound to the liver tumor tissue, even at approximately 24 hours. In addition, the dynamics of magnetic labeling from the binding of antiprobe-mediated MNPs to tumors planted on mice is similar to those of other works, ${ }^{20}$ therefore, this result is reliable. 
To compare the $\mathrm{M}$ variation using SSB with the average normalized intensity $\Delta \mathrm{I} / \mathrm{I}_{0}$ of the MRI signal, the normalized variation $\Delta \mathrm{M} / \Delta \mathrm{M}_{\max }$ of the SSB signals was employed (Figure $4 \mathrm{D}$ ). In this study, $\Delta \mathrm{M}$ was defined as the difference of $\mathrm{M}$ before and after the injection, and $\Delta \mathrm{M}_{\max }$ was $\Delta \mathrm{M}$ at 24 hours. However, $\Delta \mathrm{M} / \Delta \mathrm{M}_{\max }$ for anti-CEA MFs and MFs without antibodies was defined as 0 because both $\Delta \mathrm{M}$ and $\Delta \mathrm{M}_{\max }$ at 24 hours were considered 0 . The trends of $\Delta \mathrm{I} / \mathrm{I}_{0}$ by MRI and $\Delta \mathrm{M} / \Delta \mathrm{M}_{\text {max }}$ using SSB over time between anti-
AFP MF, anti-CEA MF, and MF without antibodies agree favorably, except for the positive and negative variation. This implied that the magnetic labeling of anti-AFP MNPs binding to liver tumors expressed the following two magnetic characteristics: the negative variation of $I$ in MRI is the distortion of the imaging field in the tumor region caused by the more superior susceptibility of MNPs than neighbor tissue, and the positive variation of $\mathrm{M}$ is the increase of $\mathrm{AC}$ susceptibility because of higher AC susceptibility of MNPs

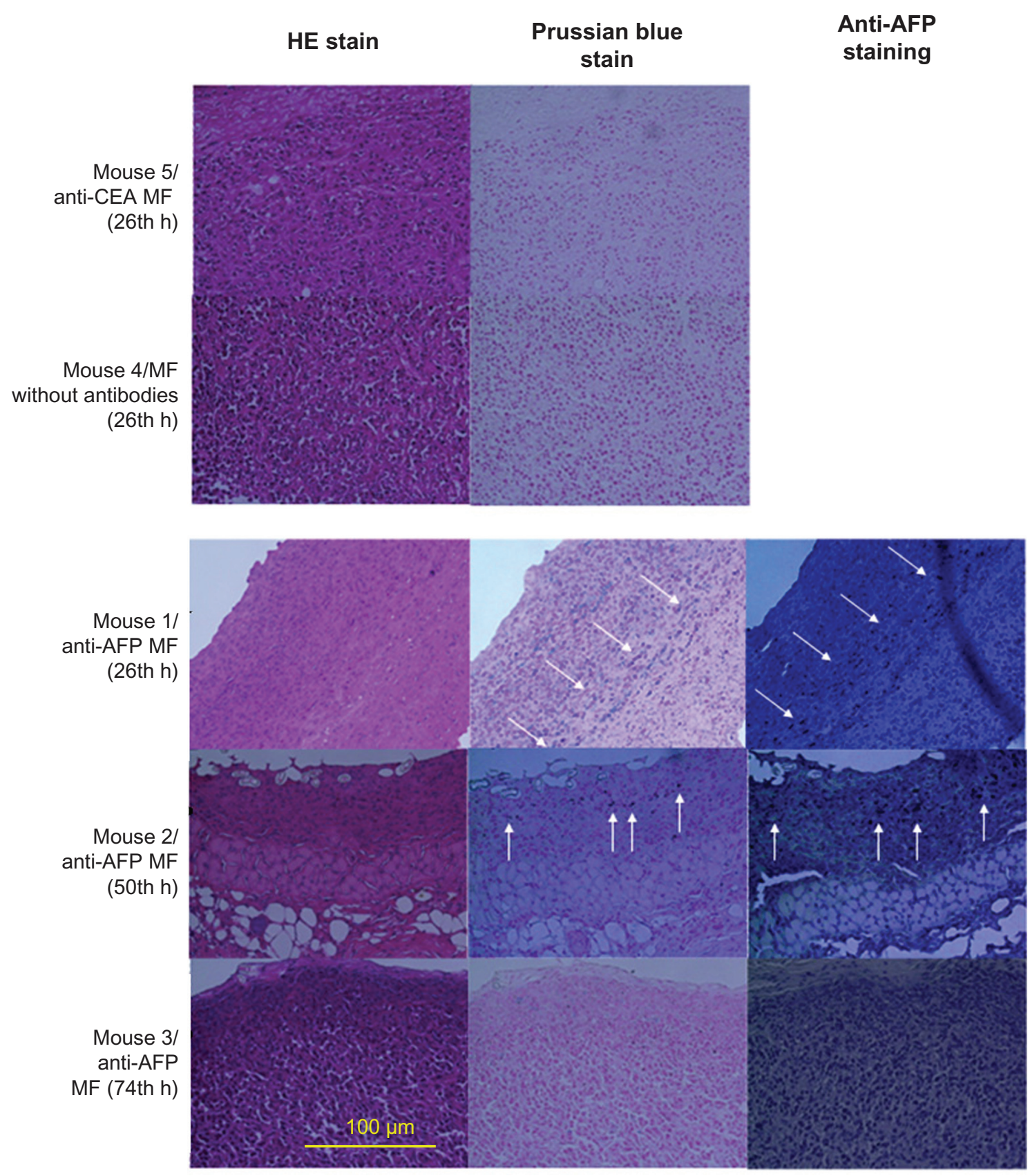

Figure $5 \mathrm{HE}$ stain and Prussian blue stain of liver tumor tissue after the injection of MFs (magnification $\times 400$ ).

Abbreviations: HE, hematoxylin and eosin; AFP, alphafetaprotein; MF, magnetic fluid; CEA, carcinoembryonic antigen. 
than neighbor tissue. Therefore, tumor examination based on magnetic labeling could be extended for not only preoperative but also intraoperative diagnosis.

\section{Biopsy tests}

HE staining showed that all the biopsies (Figure 5) were obtained from the tumor tissue by observing the morphology. For the same biopsy, the results of Prussian blue staining showed that the number of blue spots (ie, $\mathrm{Fe}^{3+}$ ions) totaled a maximum at approximately 24 hours, and decreased over time for anti-AFP MF. However, no blue spots were found for MF without antibodies and anti-CEA MF. In this study, these $\mathrm{Fe}^{3+}$ ions resulted from degraded MNPs with $\mathrm{Fe}_{3} \mathrm{O}_{4}$ material using diluted hydrochloric acid during the standard procedure of HE staining. Furthermore, the results of anti-AFP staining showed a similar distribution of brown spots to that of blue spots. The time variations in amount of brown spots and blue spots were the same. The favorable agreement between Prussian blue staining and anti-AFP staining indicates that complete MNPs with anti-AFP coating were distributed on the liver tumor tissue. In addition, the favorable agreement between the biopsy results and both magnetic examinations of MRI and SSB proves that antiAFP MNPs expressed different magnetic characteristics of magnetic labeling.

\section{Conclusion}

All the results of the in-vivo examinations of MRI and SSB and biopsy assays exhibit the same dynamics of magnetic labeling. This study verified that simple MNPs with anti-AFP coating expressed magnetic characteristics of AC susceptibility other than the distortion of the imaging field. Therefore, SSB based on the examination of AC susceptibility can be used as the instruments of intraoperative examination or other clinical requirements, extending the utilities of single magnetic modal MNPs.

\section{Acknowledgments}

This work was supported by the National Science Council, Taiwan, under grant numbers (100-2112-M-003010-), (99-2112-M-003-014-), (100-2120-M-002-015-), (100-2221-E-003-013-), (99-2112-M-002-017-MY3), and (98-2221-E-003-001-MY2), and by the Department of Health, Taiwan, under grant numbers (DOH99-TDN-111-008), (DOH100-TD-N-111-008), and (DOH101-TDN-111-004). Thank you to 7T Animal MRI Core Laboratory of the Neurobiology and Cognitive Science Center, National
Taiwan University, Taipei, Taiwan, for technical and facility support.

\section{Disclosure}

The authors report no conflicts of interest in this work.

\section{References}

1. Liao SH, Yang HC, Horng HE, Yang SY. Characterization of magnetic nanoparticles as contrast agents in magnetic resonance imaging using high-Tc superconducting quantum interference devices in microtesla magnetic fields. Supercond Sci Technol.2009;22(2):025003-1-0250035.

2. Hong CY, Wu CC, Yang SY, et al. Magnetic susceptibility reduction method for magnetically labeled immunoassay. Appl Phys Lett. 2006;88(21):212512-1-212512-3.

3. Huang KW, Yang SY, Hong YW, et al. Feasibility studies for assaying alpha-fetoprotein using antibody- activated magnetic nanoparticles. Int J Nanomed. 2012; accepted.

4. Rosensweig RE. Heating magnetic fluid with alternating magnetic field. J Magn Magn Mater. 2002;252(2002):370-374.

5. Tseng HY, Lee GB, Lee CY, et al. Localised heating of tumours utilizing injectable magnetic nanoparticles for hyperthermia cancer therapy. IET Nanobiotechnol. 2009;3(2):46-54.

6. Duguet E, Vasseur S, Mornet S, Devoisselle JM. Magnetic nanoparticles and their applications in medicine. Nanomedicine. 2006;1(2) 157-168.

7. Prijic S, Sersa G. Magnetic nanoparticles as targeted delivery systems in oncology. Radiol Oncol. 2011;45(1):1-16.

8. Nair BG, Nagaoka Y, Morimoto H, et al. Aptamer conjugated magnetic nanoparticles as nanosurgeons. Nanotechnology. 2010;21(45): 455102-1-455102-8.

9. Moritz FK, Umar M, Raymond SK, et al. A multimodal nanoparticle for preoperative magnetic delineation resonance imaging and intraoperative optical brain tumor. Cancer Res. 2003;63:8122-8125.

10. Watkin KL, McDonald MA. Multi-modal contrast agents: a first step. Acad Radiol. 2002;9(2):S285-S289.

11. Liao HS, Huang KW, Yang HC, et al. Characterization of tumors using SQUID-detected nuclear magnetic resonance and imaging. Appl Phys Lett. 2010;97(26):263701-1-263701-3.

12. Ssalamah AB, Uffmann M, Saini S, Bastati N, Herold C, Schima W Clinical value of MRI liver-specific contrast agents: a tailored examination for a confident non-invasive diagnosis of focal liver lesions. Eur Radiol. 2009;19(2):342-357.

13. Chieh JJ, Hong CY. Non-invasive and high-sensitivity scanning detection of magnetic nanoparticles in animals using high- $\mathrm{T}_{\mathrm{c}}$ scanning superconducting-quantum-interference-device biosusceptometry. Rev Sci Instrum. 2011;82(8):084301-1-084301-6.

14. Chieh JJ, Tseng WK, Horng HE, et al. In-vivo and real-time measurement of magnetic-nanoparticles distribution in animals by scanning SQUID biosusceptometry for biomedicine study. IEEE IEEE Trans Biomed Eng. 2011;58(10):2719-2724.

15. Tseng WK, Chieh JJ, Yang SY, et al. In-vivo and fast examination of iron concentration of magnetic nano-particles in an animal torso via scanning SQUID Biosusceptometry. IEEE Trans Appl Supercond. 2011;21(3):2250-2253.

16. Yang SY, Jian ZF, Horng HE, et al. Dual immobilization and magnetic manipulation of magnetic nanoparticles. J Magn Magn Mater. 2008;320(21):2688-2691.

17. Wang ZF, Stein R, Sharkey RM, et al. Carcinoembryonic antigen and alpha-fetoprotein expression and model monoclonal antibody targeting in a human hepatoma/nude. Cancer Res. 1990;50(Suppl 3): $869 \mathrm{~s}-872$ s. 
18. Horng HE, Chieh JJ, Hung KW, et al. Feasibility research on the earlystage in-vitro detection and the bio-functional MRI for liver cancer and colon cancer in animals using magnetic nanoparticles. Report of National Program on Nanotechnology supported by Department of Healthy, Executive Yuan; Republic of China; 2011.

19. Yang SY, Sun JS, Liu CH, et al. Ex vivo magnetofection with magnetic nanoparticles: a novel platform for nonviral tissue engineering. Artif Organs. 2008;32(3):195-204.
20. Qiao J, Li S, Wei L, et al. HER2 targeted molecular MR imaging using a de novo designed protein contrast agent. PLoS ONE. 2011;6(3): e18103-1-e18103-9.

\section{Publish your work in this journal}

The International Journal of Nanomedicine is an international, peerreviewed journal focusing on the application of nanotechnology in diagnostics, therapeutics, and drug delivery systems throughout the biomedical field. This journal is indexed on PubMed Central, MedLine, CAS, SciSearch ${ }^{\circledR}$, Current Contents ${ }^{\circledR} /$ Clinical Medicine,
Journal Citation Reports/Science Edition, EMBase, Scopus and the Elsevier Bibliographic databases. The manuscript management system is completely online and includes a very quick and fair peer-review system, which is all easy to use. Visit http://www.dovepress.com/ testimonials.php to read real quotes from published authors. 